\title{
The Realization of Wi-Fi Network for a Monitoring System of Building Energy Consumption
}

\author{
Liu Yunhong ${ }^{1, \text { a }}$, Liu Zuopeng ${ }^{2, \text { b }}$ \\ ${ }^{1,2}$ School of Electrical Engineering, Dalian University of Technology, Dalian, China \\ alyunhong2003@aliyun.com, 'bliuzuopeng@mail.dlut.edu.cn
}

\begin{abstract}
Keywords: building energy consumption; monitoring system; Wi-Fi wireless communication; embedded system
\end{abstract}

\begin{abstract}
With the change of energy conservation and emissions reduction situation, the universal application of building energy consumption monitoring system become increasingly important. After the current situation of public building energy monitoring analyzed, the hardware and software of energy monitoring system is optimized by the application of wireless communication technology. And then a new type of easily transformed, distributed and maintained scene information collection and communication system based on Wi-Fi is designed not only by the combination of RS485 Bus technology, Wi-Fi and internet, but by the embedded system as well. At last, a new architecture of building energy online monitoring system based on this technology is proposed.
\end{abstract}

\section{Introduction}

With the accelerating progress of urbanization in China and the increasing number of high energy consumption constructions, building energy consumption has heavily stressed on the power supply of our country ${ }^{[1]}$. There are several problems existing in domestic energy consumption monitoring system, which are mainly three aspects. First, the concept of energy consumption monitoring is obsolete, which is unable to get rid of the original one. Effective methods of analysis and prediction models are missing, so that utilization efficiency of the collected data is low. Second, the energy monitoring management system is not formed. Meanwhile, there are plenty of loopholes in the system. Third, energy monitoring system lacks the support of proven technology and funds ${ }^{[2]}$. At present, there is no equipment to meter the energy consumption of each device respectively which makes it difficult to remold the old buildings. The reasons that restrict the popularity of building energy monitoring are the difficult wiring, large amount of work and high costs.

\section{The Architecture of the System}

The building energy consumption monitoring system based on Wi-Fi consists of three parts which are information collecting module, communication module and remote server software system ${ }^{[3]}$. Information collecting module and communication module should comply with the standard communication protocols of relevant industry, which is DL/T645-1997 Communication Protocol of Multifunction Meter for watt-hour meters and CJ/T188-2004 Household Meters Data Transmission Technology Conditions for water meters, heat meters and gas meters, so all the smart meters are required to support Modbus open protocol ${ }^{[5]}$.

The energy consumption information collected by smart meters outputs to the communication module through RS485 interface and communication module transforms the information from the mode of RS485 to that of Wi-Fi wireless communication. The communication module accesses to the Internet through a wireless router and upload the information to server directly, so some hardware architectures such as the cable in the Fieldbus System and the information concentrator and coordinator in the ZigBee System can be omitted ${ }^{[5]}$. The diagram of the Wi-Fi based hardware system is shown as Fig.1. 


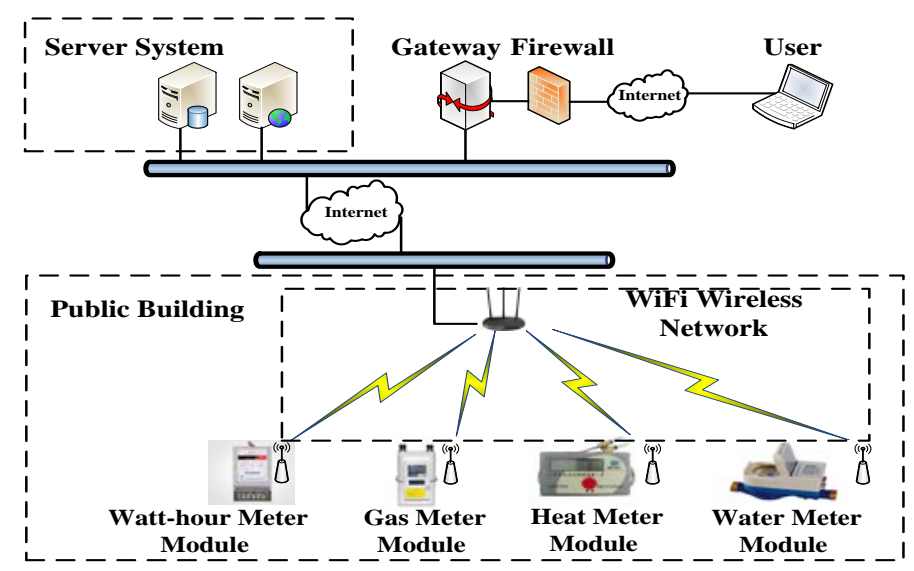

Fig.1 Hardware Structure of the Wi-Fi based Building Energy Consumption Monitoring System

Measuring terminal is integrated with both energy consumption collecting and Wi-Fi communication modules by an embedded system. Hardware system is distributed on site where the energy consumption information is collected, which consists of the smart meters of water, electricity, heat and gas, the embedded system and Wi-Fi wireless communication module.

As an example, the measurement node of electricity energy consumption hardware system is a watt-hour meter with both information collecting and communication module, and on node there is a ARM embedded system, which makes it a wireless node. The ARM single-chip microcomputer coordinates the work of automatic data collection, signal conversion and wireless communication. The structure diagram of node is shown as Fig.2.

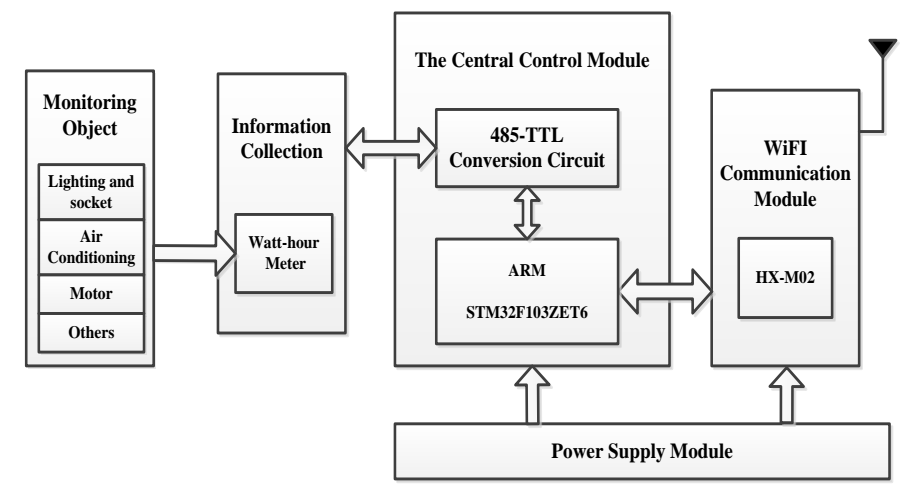

Fig.2 Structure Diagram of the Measurement Node

The Wi-Fi communication module can access the wireless router in the building after a certain settings, and then a network of information measurement and transmission can be built with multiple nodes and routers. When the wireless routers are not connected to Internet, it is an intranet and the energy consumption information would be transferred to the server in this intranet to analysis and store, and then an intranet monitoring system of building energy consumption has been set up. When the wireless routers are connected to Internet, the energy consumption information would be transferred to a networked remote server, and then it is a system that can monitor building energy consumption online and can be accessed via the Internet. The difference between two systems is the settings which in the online system is more complicated.

The Wi-Fi network used in this new type of monitoring system in public buildings is becoming more and more popular, which makes the amount of construction difficulties and tasks reduced, and saves the cost simultaneously.

\section{The Realization of Wi-Fi Wireless Communication Hardware}

The note locations are distributed in transformer, air conditioning, elevator power distribution room and some other places. The maximum communication distance can reach 100 meters indoor 
under the condition of Wi-Fi network is well built, so the communication can still be ensured without cable construction.

A module named HX-M02 is used in the communication module, which is a embedded module meeting the Wi-Fi wireless network standards. Both SPI and UART interface are integrated, and there also is a external antenna on module HX-M02. It makes the development cycle shorten that IEEE802.11 wireless network protocol stack and TCP/IP protocol stack have been built in HX-M02. The feature of low power consumption makes it possible that Wi-Fi technology can be used in the area of wireless measurement, and that of small size makes it easy to be integrated. The interface circuit diagram of HX-M02 is shown below as Fig.3

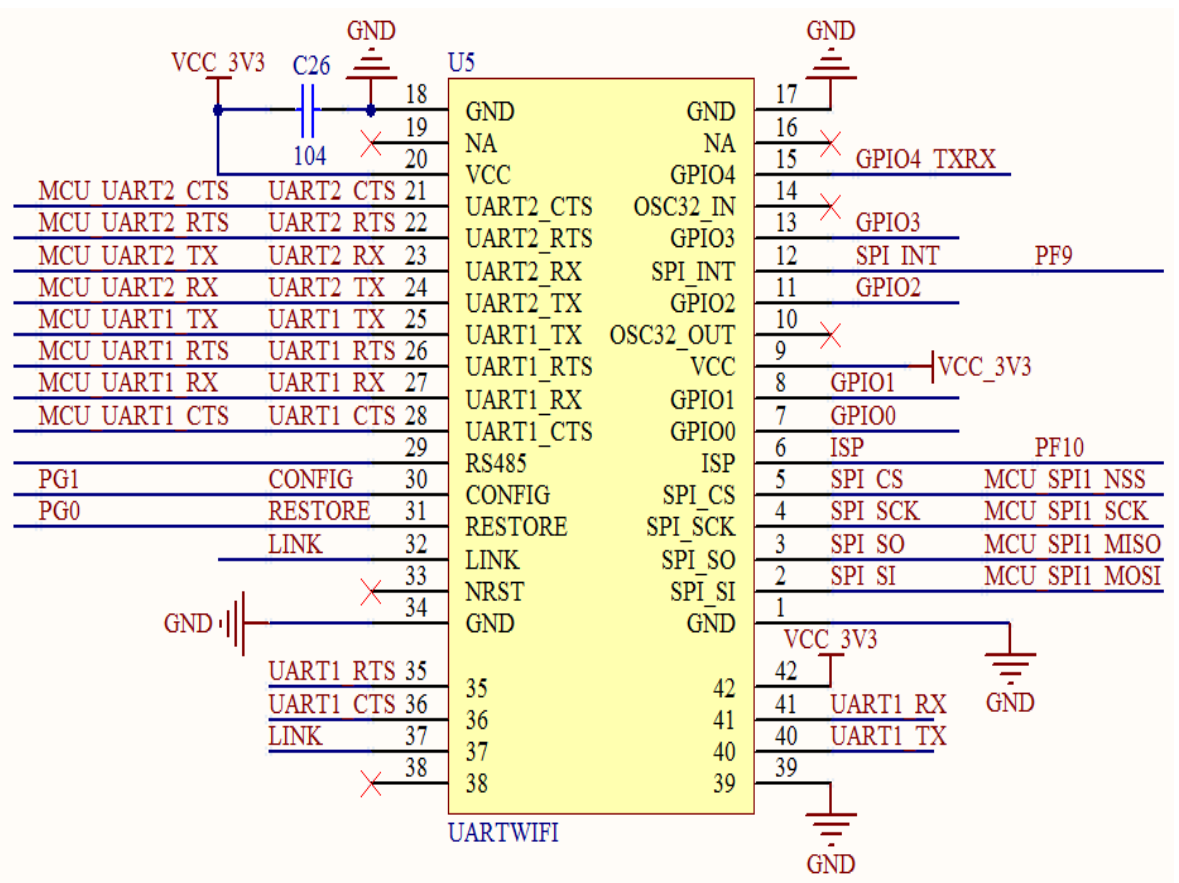

Fig.3 Interface Circuit Diagram of HX-M02

\section{The Realization of Embedded System}

The STM32 which is a 32-bit microcomputer based on ARM is the field controller in the embedded system. Because RS485 is half duplex communication mode, there is a 485-TTL conversion circuit between the ARM and the field meter, by which RS485 signal the output of smart meters can be transformed into the protocol that can communicate with ARM, and the core function of that circuit is completed by a chip named SP3485. The diagram of conversion circuit is shown as Fig.4.

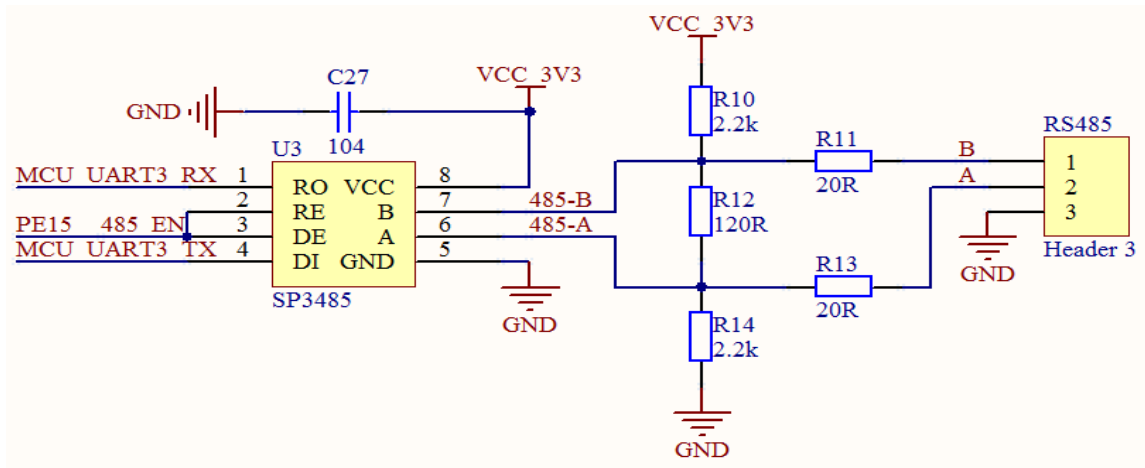

Fig.4 485-TTL Conversion Circuit 
The nodes are distributed in the location of meters, so for the node of watt-hour meter external power supply is available, but for that of the other meters a battery is necessary on the node. Low power Wi-Fi technology and hibernation design of the program make battery power Wi-Fi node reality. The design in the paper support both external and battery power supply which is $12 \mathrm{~V}$. A chip LM2596 is designed to provide a stable voltage source by reducing the voltage.

The building energy consumption information would been sent to the module HX-M02 though UART interface after that is updated from smart meter to the microcomputer which controls the module HX-M02 sent that information to the server though the Internet. Then the whole process of information collection and wireless communication is completed, and the hardware system will be in hibernation to wait for the next work cycle. The program flow chart of the ARM is shown as Fig.5.

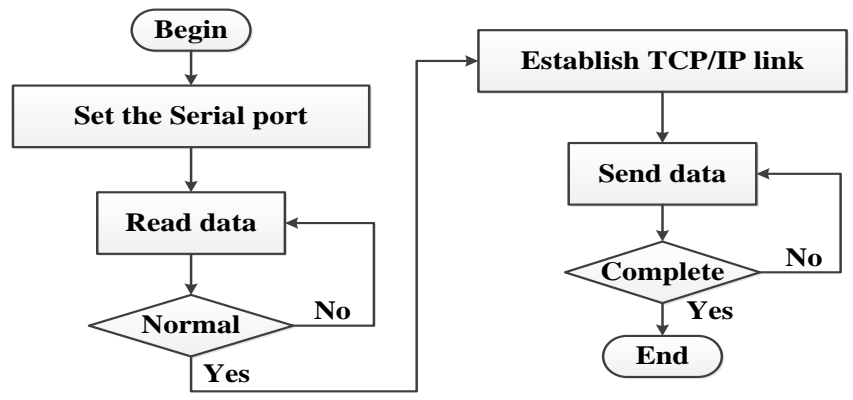

Fig.5 Program Flow Chart

\section{Summary}

Wi-Fi is a kind of mature technology, but the applications in industry is in the initial stage. It can be sure that with the development of low-power Wi-Fi and the mature technology existing, there will be more applications in industrial field. In this paper, a new type of communication combines Wi-Fi technology and RS485 has been advanced, which is used to design a new monitoring system architecture, and it is also described that how to implement both hardware and software. Combined with Internet technology and database technology, a remote monitoring system of building energy consumption has been realized, and this wireless communication plan has been used and tested in the energy consumption monitoring system of iLab remote experimental center of Dalian University of Technology, and the system is running well.

\section{References}

[1] C.Y. Wei, Y.Z. Li: Design of energy consumption monitoring and energy-saving management system of intelligent building based on the Internet of things, International Conference on Electronics, Communications and Control (ICECC). (2011).

[2] Y.P. Chen, X.M. Mu, J.L. Zhang and Z. Lu: Development of Monitoring System of Building Energy Consumption, International Forum on Computer Science-Technology and Applications. (2009)

[3] S.Z. Sanche, R.M. Fernandez-Cantf, etc: Monitoring and Remote Control of Energy Consumption by Wi-Fi Networks, Multi-Conference on Systems, Signals \& Devices (SSD), (2014).

[4] C.A. Trasvina-Moreno, A. Asensio, R. Casas, R. Blasco and A. Marco: Wi-Fi Sensor Networks: A study of energy consumption, Multi-Conference on Systems, Signals \& Devices (SSD), (2014).

[5] C.W. Yan and J. Yao: Research on the optimization of building energy efficient measures based on incremental costs, 2nd International Conference on Computer Engineering and Technology (ICCET), (2010) 